\title{
Incidental development of associations in simultaneous learning
}

\author{
BENTON J. UNDERWOOD \\ Northwestern University, Evanston, Illinois 60201 \\ and \\ ARNOLD M. LUND \\ Bell Laboratories, Holmdel, New Jersey 07733
}

\begin{abstract}
Subjects learned two lists of words simultaneously by free recall procedures. The purpose was to determine if the words from the two different lists occurring together on the study trials became associated as a result of their contiguous occurrence. Tests of incidental learning were made by having the subjects learn a bidirectional paired associate list in which the pairs consisted of appropriate pairings (words that occurred together on the study trials of free recall) or inappropriate pairings (words that had not occurred together during free recall learning). Appropriate pairings resulted in heavy positive transfer, whereas inappropriate pairings produced severe negative transfer. The evidence seemed to indicate that associations were formed incidentally between words appearing together during the study trials of free recall.
\end{abstract}

A full understanding of the conditions under which associations are established remains a fundamental problem in human learning. One factor that may be involved in the formation of associations, a factor that has been studied over many decades, is contiguity. Recently, Glenberg and Bradley (1979) reached the conclusion that mere contiguity of two words emitted by a subject does not result in the formation of an association between those two words. In the present experiment, we have used simultaneous learning as a device for producing contiguity between two items without any necessity of the subjects' intentionally trying to associate the two. In simultaneous learning, two or more lists are presented for learning at once, with the words from the two or more lists being shown together during study. The empirical question is whether or not associations develop between two items from two different lists being studied simultaneously.

The logic of the experiment required two stages, one in which simultaneous learning occurred and during which associations could develop, and a second stage in which a test was made for associative learning that may have occurred during the first stage. We chose to use a paired associate list to test for associative learning; the

This research was supported by the Personnel and Training Research Programs, Psychological Sciences Division, Office of Naval Research, under Contract N00014-78-C-0661, Contract Authority Identification NR $154-424$. Reproduction in whole or in part is permitted for any purpose of the United States government. Requests for reprints should be sent to Benton J. Underwood, Department of Psychology, Northwestern University, Evanston, Illinois 60201 . items in the list had previously been a part of the simultaneous learning of two lists by free recall.

\section{METHOD}

\section{Lists}

The two lists used in simultaneous learning consisted of 16 four-letter nouns typed in capital letters and 16 nouns of at least two syllables typed in lower case. The paired associate list contained 16 pairs; each pair consisted of one short and one long noun. The paired associate list was presented for bidirectional learning; that is, each word in a pair occurred as the stimulus term half the time and as the response term half the time. A single paired associate list was used, and the differences in the transfer paradigms were produced by varying the pairings of the word during simultaneous learning.

\section{Conditions}

All subjects had five study-test trials of free recall simultaneous learning prior to paired associate learning. On a free recall study trial, one word from each of the two lists occurred together for $4 \mathrm{sec}$, then another pair (one word from each list) for another $4 \mathrm{sec}$, and so on. The subjects were instructed to learn the two lists separately; the words in the lists were identified by their lengths (long or short).

There were four conditions, differing in the nature of the pairings as they occurred in simultaneous learning. In Condition AP (appropriately paired), the pairings of the words in the test list (the paired associate list) were exactly the same as the pairings in simultaneous learning. If the two words occurring together in simultaneous learning become associated, positive transfer should occur on the test list for this condition. Condition IP (inappropriately paired) was a contrast to Condition AP in that the two words occurring together in simultaneous learning were never paired on the test list. If associations develop in simultaneous learning, they should produce interference as the subjects try to learn the test list in Condition IP. Condition VP (variably paired) was another potential interference condition. 
On the five simultaneous learning trials, the pairings of the long and short words differed on each of the trials, and a sixth pairing occurred on the test list. Finally, In Condition C (control), the subjects were given simultaneous learning on two lists made up of long and short nouns, but none of these words occurred on the test list. This condition served as a control for nonspecific transfer only.

\section{Procedure and Subjects}

As noted earlier, the lists for simultaneous learning were presented at a $4-\mathrm{sec}$ rate on the study trial, with the order of the pairs differing on each of the five trials. The list of short nouns was always recalled before the list of long nouns; $60 \mathrm{sec}$ was allowed for the recall of each list on each trial. The paired associate (test) list was presented for one study and eight anticipation trials at $3: 3$-sec rate. On any given trial, half the stimulus terms were the short words and half were the long words. Across the trials, each word in a pair served equally often as the stimulus term and as the response term. The pairs were presented in four different orders. The subjects were fully informed about the bidirectional learning of the test list.

Four groups of 20 subjects each (college students) represented the four conditions. Subjects were assigned to conditions from a block-randomized schedule.

\section{RESULTS AND DISCUSSION}

\section{Simultaneous Learning}

There were five trials of simultaneous learning. The mean numbers correct on the fifth trial varied from 9.70 to 12.50 for the various lists viewed individually. In only five cases out of a possible 160 (80 subjects, each having two lists for simultaneous learning) did the subject get all 16 items correct from one of the lists of the fifth trial. We mention these matters to indicate that simultaneous learning did not reach a high level in the five trials.

\section{Transfer to Paired Associates}

The mean number of correct responses by trials for the paired associate test list is shown in Figure 1. As can be seen, the differences in transfer were large. Heavy positive transfer occurred in Condition AP, but in fact the amount is underestimated because of a ceiling effect. Seven of the 20 subjects responded correctly to all stimuli on the first anticipation trial. The performance under Condition $\mathrm{C}$ was inferior to that under Condition AP, but it is difficult to judge the exact magnitude of the true difference. There are two reasons for this. First, there is the ceiling problem for Condition AP, as just noted; this indicates that we are underestimating the amount of positive transfer. Second, there is the fact that for Condition $\mathrm{C}$ it was necessary for the subjects to learn the items per se in the test list, whereas that was not true for the other conditions. The performance under Condition $\mathrm{C}$ is some unknown amount lower than would have been the case if item learning had not been required. The subjects in the other conditions, of course, learned most of the items making up the paired associate list when they had simultaneous learning. Still, it seems quite unlikely that if we knew the true differences we

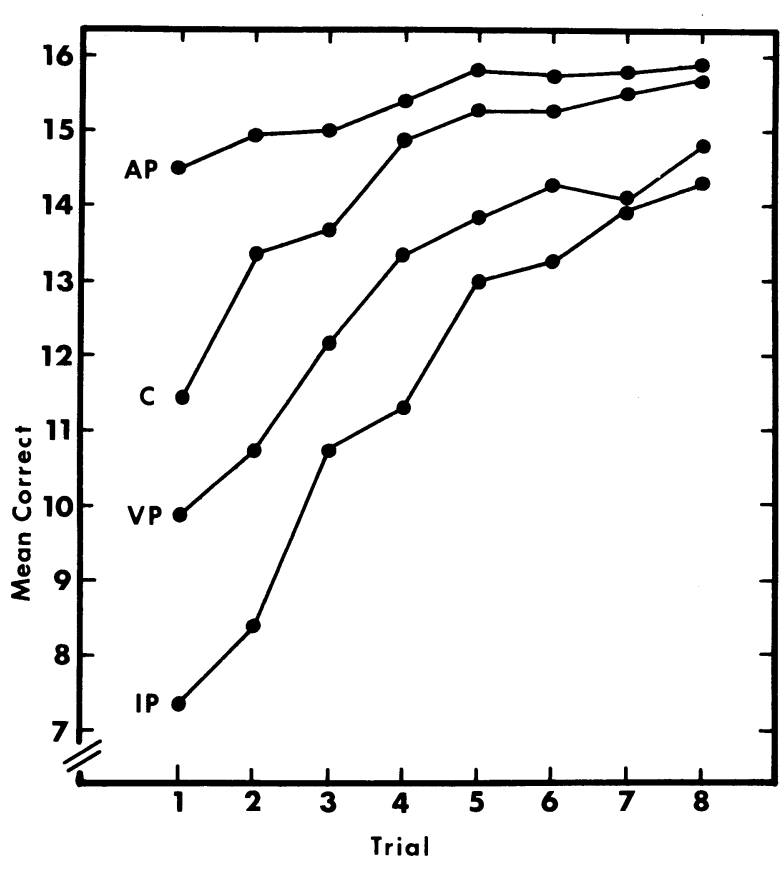

Figure 1. Transfer from simultaneous learning to paired associate learning. See text for description of conditions.

would conclude that there was no positive transfer in Condition AP.

The differences among the four conditions were reliable when the mean total correct across eight trials was used as the response measure $[\mathrm{F}(3,76)=11.13$, $\mathrm{MSe}=323.86]$ and when the mean number correct on the first anticipation trial was used $[F(3,76)=13.40$, $\mathrm{MSe}=13.35]$. Each condition differed reliably from each adjacent condition either on the mean total correct or on the mean correct on the first trial.

Negative transfer was produced by Conditions VP and IP. The fact that the interference was somewhat greater for Condition IP than for Condition VP indicates that a single strong associate will produce more negative transfer than several weak associates. Intrusions in the test list reflecting the pairings that occurred in simultaneous learning were numerous in Condition IP, averaging 7.15 for the 20 subjects.

The above evidence leaves no doubt that associations develop between the two words presented together in simultaneous learning, and this is true even if the words are presented together only once. The question that immediately arises concerns the degree of awareness of the subjects in the growth of the associations. Did the subjects deliberately set about to learn the associations or did these associations develop incidentally?

If subjects deliberately tried to associate the items occurring together in simultaneous learning, they might do so in order to use one word as a cue to produce the other during free recall. If this is true, then it is very likely that the output order for the two lists (making 
up the simultaneous learning task) should be about the same. We made a determination of the relationship between the output orders of the two lists for Conditions AP, IP, and $\mathrm{C}$ on the fifth (last) simultaneous learning trial. The relationship was expressed by a rankorder correlation for each subject. Of course, these correlations could be calculated only for the items that occurred together in simultaneous learning and were also recalled. Out of the 60 subjects, we found only two cases in which intentional learning would be clearly indicated. In one case, 16 items from both lists were recalled and the order of output for the two lists was identical. This will be illustrated. Let $\mathrm{Ab}, \mathrm{Cd}, \mathrm{Ef}$, and $\mathrm{Gh}$ represent 4 of the 16 sets of words occurring in simultaneous learning. If in the recall of the four-letter words the output order was E, C, G, and A and if it was also found that the output order for the long words was $f, d, h$, and $b$, the two output orders were said to be correlated perfectly for this subject. The same correlation was found for a second subject, who recalled 13 items from each list.

The above two cases were the exceptions. Out of the 60 cases, 26 of the correlations were negative and 34 were positive, indicating that overall there was very little relationship between the output orders of the two lists making up the simultaneous task. In turn, this suggests that associations were not learned intentionally in order to use a cuing procedure at recall. It is logically possible, of course, to have associated items used as cues for each other without having the output order of the two lists correlated, but this does not seem likely. It was also determined that the number of times each item was given correctly during simultaneous learning of one of the lists was not correlated with the number of times the item with which it was paired was given correctly. Out of the 60 cases, 23 of the correlations were negative and 37 were positive. The average correlation was .10. Again, while these data are not definitive on the matter, they do not indicate that subjects set about to associate the items in order to use the associations to cue the items at recall.

One other finding should be reported. The subjects in Condition $\mathbf{C}$ provide a test of the underlying similarities between simultaneous learning of free recall lists and paired associate learning. It will be remembered that in this condition the words used for simultaneous learning were completely different from those used in the test list. The correlation between the total number correct for the two tasks across the 20 subjects was .21 , far below an acceptable level of statistical significance.

This experiment gave a clear answer to the question prompting it. Items occurring together in simultaneous learning do get associated, and strongly so. The amount of negative transfer observed in Condition IP in Figure 1 represents far more negative transfer than is usually found in an $\mathrm{A}-\mathrm{Br}$ paradigm in the usual two-list transfer situation. In that situation, A-B is deliberately learned to a given level prior to learning a second list that is formed by repairing the stimulus and response terms of the first list. In the present study, the evidence indicates that most subjects did not deliberately try to associate the two words occurring together in simultaneous learning. This may be a key to an account of the large transfer effects. During the test-list learning, the subjects would necessarily try to activate situational associations to the stimulus terms. It may be that the subjects were simply not aware of the fact that they had associated items in simultaneous learning, and hence they had a difficult time discriminating between the associations learned incidentally and those learned intentionally in the test list. This lack of discrimination is suggested by the intrusion data, data that are particularly sensitive to breakdowns in discrimination.

As noted earlier, there are recent experiments (e.g., Glenberg \& Bradley, 1979) that show that associations do not develop as a result of the mere contiguity of two words. Nevertheless, if a subject is deliberately trying to learn a central task per the instructions given him by the experimenter, incidental learning of contiguously occurring items having nothing to do with the central learning task may well occur. This type of incidental learning seems to occur in verbal discrimination learning (e.g., Zechmeister \& Underwood, 1969), and it seems to have occurred here in simultaneous learning.

\section{REFERENCES}

Glenberg, A. M., \& Bradley, M. M. Mental contiguity. Journal of Experimental Psychology: Human Learning and Memory, 1979, 5, 88-97.

Zechmeister, E. B., \& Underwood, B. J. Acquisition of items and associations in verbal discrimination learning as a function of level of practice. Journal of Experimental Psychology, 1969, 81, 355-359.

(Received for publication November 12, 1980.) 